\title{
A Double-Guidewire Technique to Facilitate Deep Cannulation and Stenting in a Patient With Altered Gastrointestinal Anatomy
}

\author{
Hideaki Kawabata ${ }^{\mathrm{a}, \mathrm{b}}$, Yuki Ueda ${ }^{\mathrm{a}}$, Yuji Okazaki ${ }^{\mathrm{a}}$, Misuzu Hitomi ${ }^{\mathrm{a}}$, \\ Katsutoshi Yamaguchi ${ }^{a}$, Yukino Kawakatsu ${ }^{\mathrm{a}}$, Masatoshi Miyata ${ }^{\mathrm{a}}$, \\ Shigehiro Motoi ${ }^{\mathrm{a}}$
}

\begin{abstract}
Endoscopic retrograde cholangiopancreatography (ERCP) in patients with altered gastrointestinal (GI) anatomy remains a challenging procedure. We experience particular difficulty passing a catheter and devices in patients who have biliary stricture or severe deformity, despite successful biliary guiding. A 70-year-old man with Roux-en-Y reconstruction for gastric cancer suffered pancreatic head cancer with biliary stricture. Seven months after biliary stenting, he developed acute occlusive suppressive cholangitis due to stent occlusion. We extracted the stent and inserted a guidewire into the intrahepatic bile duct. However, we were unable to pass the catheter due to severe stricture of the lower common bile duct and axis-dismatch between the bile duct and the catheter. After inserting another of the same guidewire into the bile duct, we were able to easily pass the catheter into the bile duct, resulting in the successful insertion of a new plastic stent. A double-guidewire technique is useful and worth attempting for the deep insertion of devices through biliary strictures in patients with an altered GI anatomy.
\end{abstract}

Keywords: Double-guidewire; Altered gastrointestinal anatomy; Endoscopic retrograde cholangiopancreatography

\section{Introduction}

Endoscopic retrograde cholangiopancreatography (ERCP) in patients with altered gastrointestinal (GI) anatomy remains a challenging procedure despite the development of various devices and techniques for its performance $[1,2]$. One technical

Manuscript submitted November 26, 2018, accepted December 3, 2018

aDepartment of Gastroenterology, Kyoto Okamoto Memorial Hospital, Kyoto, Japan

bCorresponding Author: Hideaki Kawabata, 58 Nishinokuchi, Sayama, Kumiyama-cho, Kuze-gun, Kyoto 613-0034, Japan.

Email: hkawabata@okamoto-hp.or.jp

doi: https://doi.org/10.14740/jmc3220 difficulty is the cannulation of the biliary orifice from an altered position. We often need to adjust and optimize the angle of approach in difficult situations with postoperative adhesion and deformity using forward-viewing endoscopes without an elevator system [3, 4]. Reported success rates of cannulation when using a forward-viewing endoscope in Billroth II anatomy are only $81-87 \%[5,6]$. We experience particular difficulty passing a catheter and devices in patients who have biliary stricture or severe deformity, although biliary guiding is successfully achieved, because it is difficult to exert effective force on a catheter or device due to axis-mismatch and the instability of the endoscope.

We herein report a case using a double-guidewire technique to facilitate deep cannulation and stenting for biliary stricture in patients with altered GI anatomy.

\section{Case Report}

A 70-year-old man underwent total gastrectomy with Rouxen-Y reconstruction for gastric cancer in January 2016. Computed tomography revealed a low-density mass with dilatation of the biliary tract in February 2018 (Fig. 1a). ERCP showed a bending, meandering dilatation and a lower stricture of the common bile duct (Fig. 1b), which was pathologically diagnosed as adenocarcinoma. Endoscopic sphincterotomy and insertion of a 5-Fr plastic stent (Zimmon Biliary Stent; Cook Medical, Tokyo, Japan) was performed. The patient then started chemotherapy with gemcitabine and nabpaclitaxel.

In September 2018, he developed acute occlusive suppressive cholangitis due to stent occlusion. Therefore, we attempted to exchange the plastic stent. We reached and identified the major papilla that was swollen due to tumor invasion using a colonoscope (PCF-Q260AZI; Olympus Co., Ltd, Tokyo, Japan). First, we tried to cannulate alongside the plastic stent using a wire-guided technique (VisiGlide 2; Olympus Co., Ltd.; and MTW; MTW Endoskopie, Wesel, Germany). However, it was impossible to optimize the angle of approach. We then extracted the plastic stent and inserted a guidewire into the intrahepatic bile duct (Fig. 2a). However, we were unable to pass the catheter due to severe stricture of the lower 


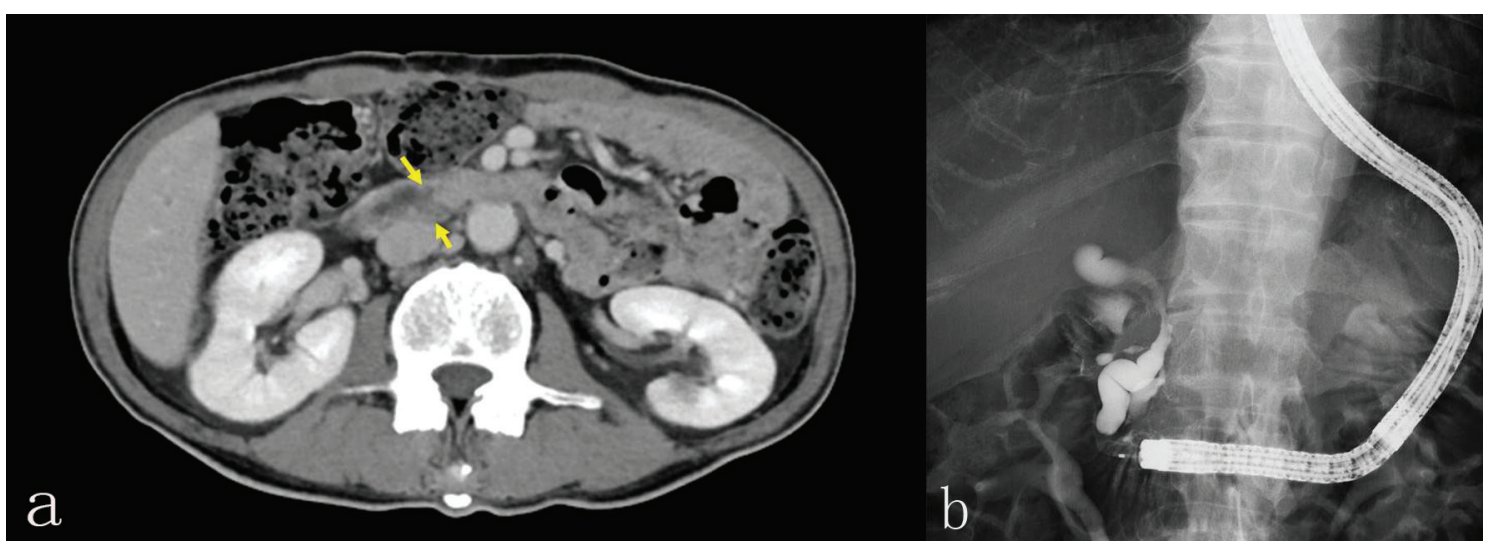

Figure 1. (a) Computed tomography revealed a low-density mass with dilatation of the biliary tract. (b) ERCP showed a bending, meandering dilatation and a lower stricture of the common bile duct.

common bile duct and axis-dismatch between the bile duct and the catheter (Fig. 2b). Therefore, we inserted another of the same guidewire into the bile duct (Fig. 3a, b) and were able to easily pass the catheter into the bile duct (Fig. 3c), resulting in the successful insertion 7-Fr plastic stent (Zimmon Biliary Stent; Cook Medical) (Fig. 3d). No procedure-related complications were observed, and the postoperative course was uneventful.

\section{Discussion}

We were able to pass a catheter through a malignant biliary stricture and insert a plastic stent with the assistance of a double-guidewire technique in a patient with altered GI anatomy. A double-guidewire can reduce the curvature of the guidewire, straighten the axis and stabilize the endoscope, making it easier to exert direct, effective force on a catheter or devices. To our knowledge, this is the first report showing the utility of a double-guidewire technique for deep insertion of devices while approaching the papilla from the caudal direction.

Although several devices, including rotatable sphincterotomes, a bendable-tip cannula and an S-shaped sphincterotome, have been developed to facilitate cannulation [1], devices to assist deep cannulation in patients with biliary stricture have not yet been reported. This double-guidewire technique requires no preparation of any special devices and is easily performed, as we can insert the second guidewire alongside the first one. Furthermore, it should be possible to use this technique during stenting for GI stricture and endoscopic ultrasound (EUS)-guided procedures.

In conclusion, this double-guidewire technique is useful and worth attempting for the deep insertion of a catheter and devices through biliary strictures in patients with an altered GI

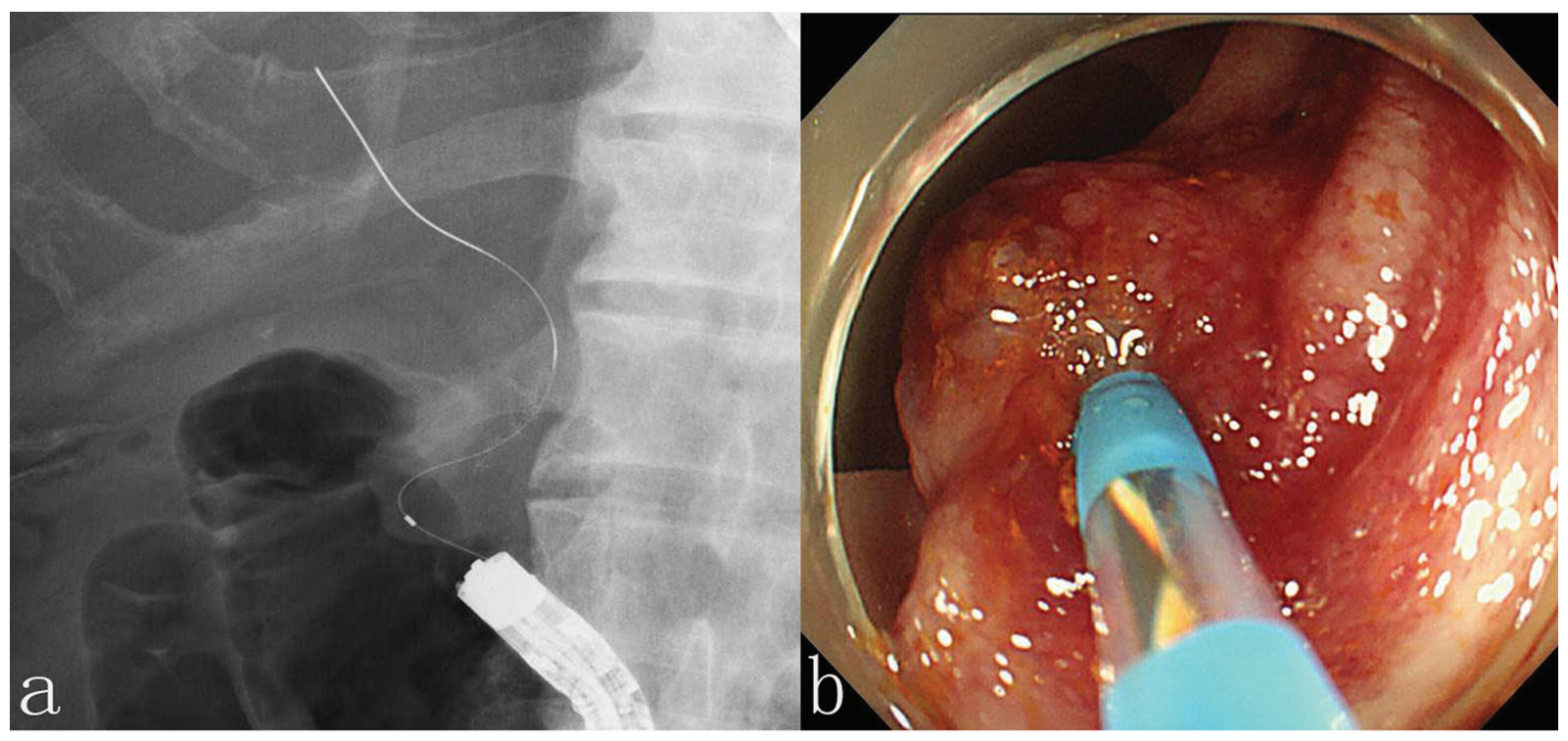

Figure 2. (a) A guidewire (VisiGlide 2; Olympus Co., Ltd, Tokyo, Japan) was inserted into the intrahepatic bile duct. (b) A catheter (MTW; MTW Endoskopie, Wesel, Germany) was not passed due to severe stricture of the lower common bile duct and axisdismatch between the bile duct and the catheter. 

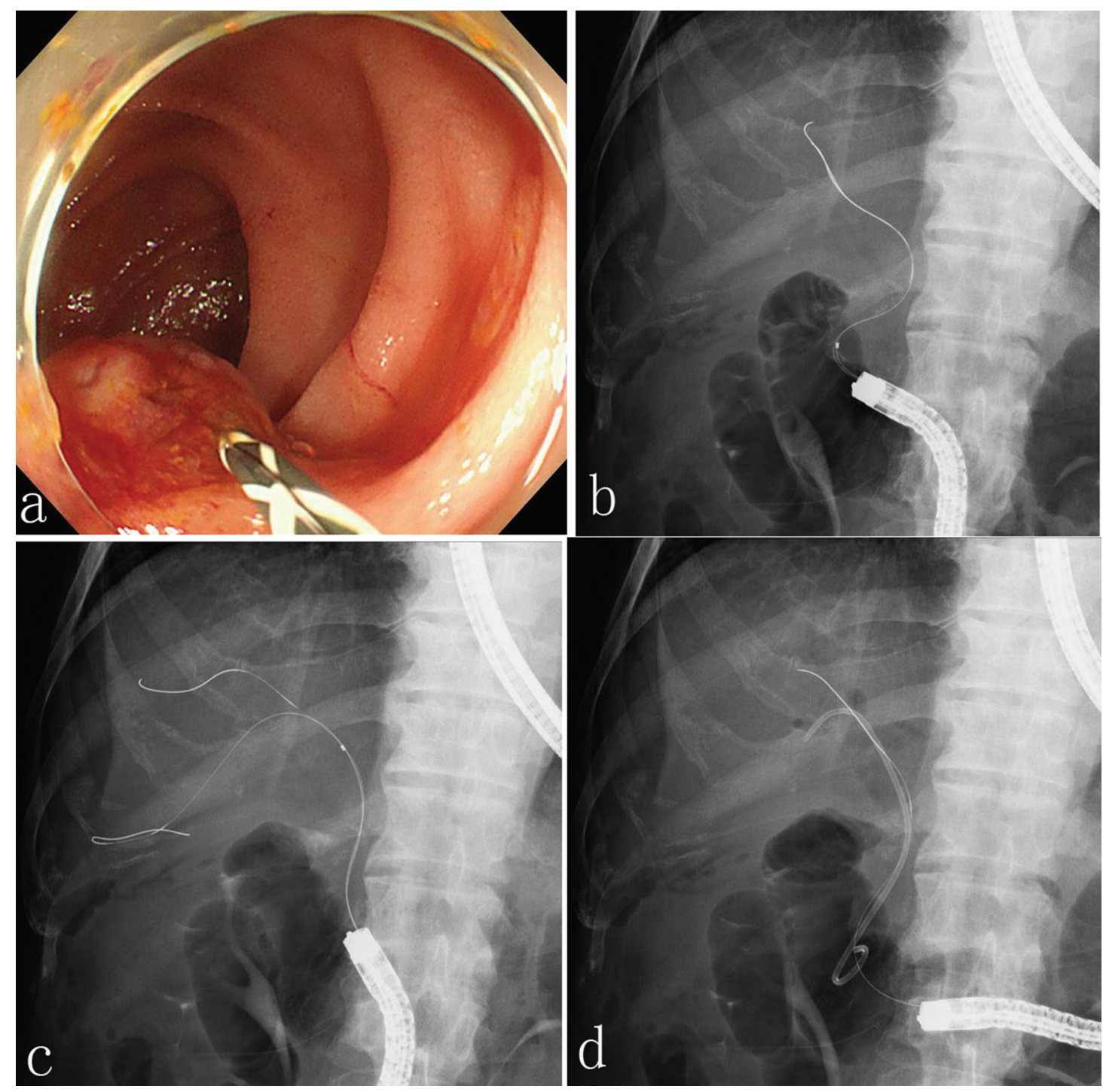

Figure 3. (a, b) Another of the same guidewire was inserted into the bile duct. (c) The catheter was easily passed into the bile duct. (d) A 7-Fr plastic stent (Zimmon Biliary Stent; Cook Medical) was successfully inserted.

anatomy. More experience is needed to confirm its utility.

\section{Conflict of Interest}

The authors declare that they have no conflict of interest.

\section{References}

1. Committee AT, Enestvedt BK, Kothari S, Pannala R, Yang J, Fujii-Lau LL, Hwang JH, et al. Devices and techniques for ERCP in the surgically altered GI tract. Gastrointest Endosc. 2016;83(6):1061-1075.

2. Moreels TG. Altered anatomy: enteroscopy and ERCP procedure. Best Pract Res Clin Gastroenterol. 2012;26(3):347357.
3. Lee A, Shah JN. Endoscopic approach to the bile duct in the patient with surgically altered anatomy. Gastrointest Endosc Clin N Am. 2013;23(2):483-504.

4. Okabe Y, Ishida Y, Kuraoka K, Ushijima T, Tsuruta O. Endoscopic bile duct and/or pancreatic duct cannulation technique for patients with surgically altered gastrointestinal anatomy. Dig Endosc. 2014;26(Suppl 2):122126.

5. Lin LF, Siauw CP, Ho KS, Tung JC. ERCP in postBillroth II gastrectomy patients: emphasis on technique. Am J Gastroenterol. 1999;94(1):144-148.

6. Kim MH, Lee SK, Lee MH, Myung SJ, Yoo BM, Seo DW, Min YI. Endoscopic retrograde cholangiopancreatography and needle-knife sphincterotomy in patients with Billroth II gastrectomy: a comparative study of the forward-viewing endoscope and the side-viewing duodenoscope. Endoscopy. 1997;29(2):82-85. 